\title{
КЛИНИЧЕСКИЙ СЛУЧАЙ ПОЗДНЕЙ ДИАГНОСТИКИ ПЕРВИЧНОГО ГИПЕРПАРАТИРЕОЗА
}

\author{
Пахахинова С.В., Молонова Л.М., Нимаева Д.Э.
}

ГАУЗ «РКБ им. Семашко» МЗ Республики Бурятия, Улан-Удэ.

Первичный гиперпаратиреоз (ПГПТ) - заболевание, характеризующееся избыточной секрецией паратгормона при верхне-нормальном или повышенном уровне кальция крови вследствие первичной патологии околощитовидных желез. В 85-90\% случаев - солитарная аденома околощитовидных желез, в 5-10\% - множественные аденомы или гиперплазия нескольких/всех околощитовидных желез, в $1 \%$ рак околощитовидных желез. ПГПТ может встречаться во всех возрастных группах, средний возраст составляет 54-59 лет.

ЦЕЛЬ: привлечь внимание специалистов первичного звена и организаторов здравоохранения к проблеме ранней диагностики ПГПТ.

МАТЕРИАЛЫ И МЕТОДЫ: представлен катамнез заболевания пациентки Д. с ПГПТ.

Пациентка Д., 60 лет, поступила в августе 2018г в отделение эндокринологии с жалобами на: боли в грудной клетке, в крупных суставах, в грудном отделе позвоночника, деформацию грудной клетки, уменьшение роста на 14 см за 3 года ;

Считает себя больной с 2015 г., когда после травмы (упала с высоты роста) постепенно стала отмечать вышеперечисленные жалобы. При обследовании у ревматолога выставлен диагноз: «Псориатический артрит». По результатам МСКТ позвоночника от 06.2016г: Остеохондроз грудно-поясничного отдела позвоночника. Спондилоартроз. Диффузный остеопороз. Клиновидные деформации тел Th11, Th12 позвонков. Принимала бисфосфонаты, препараты кальция. Наблюдалась у ревматолога, гематолога (подозрение на миеломную болезнь). К эндокринологу не направлялась. В августе 2016 г. получила закрытый перелом шейки левой бедренной кости. Прошла денситометрию в декабре 2017 г., выявлено снижение T-критерия: в L1-4 до -3,4, в бедренной кости слева до -3,9, справа до -2,6. B январе 2018г впервые консультирована эндокринологом, направлена на обследование. На повторный прием к эндокринологу с результатом паратгормона (3484 пг/мл $(\mathrm{N}=15-65)$ ) не пришла, в связи с полученным переломом хирургической шейки левой плечевой кости от 01.2018 г. Продолжала принимать бисфосфонаты. В июне 2018 г сдала анализы: ПТГ - 1703 пг/мл, витамин D - 23,48 нг/мл (N=30-80), кальций ионизированный 1,83 ммоль/л (N=1,15-1,29). В августе 2018 г. госпитализирована в отделение эндокринологии. При обследовании: ПТГ: 1132 пг/мл; Витамин D: 31,9 нг/мл; Са ионизированный: 2 мм/л, общий кальций: 3,14 мм/л $(\mathrm{N}=2,15-2,5)$; неорганический фосфор: 0,78 мм/л $(\mathrm{N}=0,81-1,45)$. Проведено: МСКТ мягких тканей шеи, выявлено правостороннее паращитовидно-паратрахеальное объемное образование правильной формы, с четкими ровными контурами, размерами до 55×28×21 мм. Образование тесно прилегает к щитовидной железе, с наличием относительно четкой ровной границы между ними. УзИ почек: в нижних сегментах синуса обеих почек микролиты 0,4см). Выписана с диагнозом: ПГПТ, костно-висцеральная форма. Правосторонняя аденома паращитовидной железы. Генерализованный остеопороз смешанного генеза (постменопаузальный и вследствие гиперпаратиреоза) тяжелой степени. В сентябре 2018г проведена селективная паратэктомия справа. Анализы при контроле: ПтГ во время операции: 1315 пг/мл; после операции через 24 часа: 151,4 пг/мл, общий кальций: 2,18 мм/л. Послеоперационная гистология: аденома паращитовидной железы смешанного строения.

ВЫводы: при своевременной диагностике ПГПт современные возможности лечения позволяют прогнозировать благоприятный исход заболевания. 